\title{
QUASIANALYTIC FUNCTIONALS AND PROJECTIVE DESCRIPTIONS
}

\author{
JOSÉ BONET and REINHOLD MEISE*
}

(To our friend Dietmar Vogt on the occasion of his 60th birthday)

\begin{abstract}
The topology of the weighted inductive limit of Fréchet spaces of entire functions in $N$ variables which is obtained as the Fourier Laplace transform of the space of quasianalytic functionals on an open convex subset of $\mathbf{R}^{N}$ cannot be described by means of weighted sup-seminorms.
\end{abstract}

\section{Introduction}

Let $\mathscr{A}\left(\mathrm{R}^{N}\right)$ denote the space of all real analytic functions on $\mathrm{R}^{N}$ with complex values. Its strong dual $\mathscr{A}\left(\mathbf{R}^{N}\right)_{b}^{\prime}$ is isomorphic to an (LF)-space $\mathscr{F} \mathscr{A}^{\prime}\left(\mathbf{R}^{N}\right)$ of entire functions on $C^{N}$ via Fourier Laplace transform. Each step space of this (LF)-space is a Fréchet space whose topology is given by weighted sup-norms. Ehrenpreis [9] showed that the topology of $\mathscr{F} \mathscr{A}^{\prime}\left(\mathbf{R}^{N}\right)$ cannot be described by weighted sup-seminorms. Similar questions on the projective description were later investigated by various authors (see Bierstedt [1]).

In the present paper this question is investigated for the spaces $\mathscr{F}_{\mathscr{E}} \mathscr{E}_{\{\omega\}}^{\prime}(G)$ of entire functions on $\mathrm{C}^{N}$ which arise as the Fourier Laplace transforms of $\mathscr{E}_{\{\omega\}}(G)_{b}^{\prime}$, where $G$ is a convex open set in $\mathrm{R}^{N}$ and $\mathscr{E}_{\{\omega\}}(G)$ denotes the space of all $\omega$-ultradifferentiable functions of Roumieu type on $G$ for a given weight function $\omega$ (see 2.1). When $\omega$ is a non-quasianalytic weight function, we showed in [4] that the natural (LF)-topology on $\mathscr{F}^{\mathscr{E}}\{(\omega\})$ cannot be given by weighted sup-seminorms. Now we prove that this result also holds when $\omega$ is quasianalytic (see Theorem 3.1). To do this we have to use arguments which are quite different from those that we applied in [4], because they were based on the fact that $\mathscr{E}_{\{\omega\}}(G)$ contains non-trivial functions with compact support.

The proof of our main result (Theorem 3.1) relies on the observation that Theorem 3.1 holds if for each convex open set $G$ in $\mathrm{R}^{N}$ there exists $\mu \in$ $\mathscr{E}_{\{\omega\}}\left(\mathrm{R}^{N}\right)^{\prime}$ having special properties (see Lemma 3.2) such that the convolution \footnotetext{
2670.

* The research of J. Bonet was partially supported by MCYT and FEDER, Project no. BFM2001-

Received December 18, 2002.
} 
operator $T_{\mu}$ induced by $\mu$ maps $\mathscr{E}_{\{\omega\}}(G)$ into itself and is not surjective. For $N=1$ the existence of such a quasianalytic functional $\mu$ can be shown by modifying a construction in Braun, Meise, and Vogt [8]. The case $N>1$ is then reduced to the case $N=1$ by elementary geometric considerations.

\section{Notation and Preliminaries}

In this preliminary section we introduce the notation that will be used throughout the paper. By $|\cdot|$ we denote the Euclidean norm on $\mathrm{C}^{N}, N \in \mathrm{N}$, while for $a \in \mathrm{C}^{N}$ and $r>0$ we let $B(a, r):=\left\{z \in \mathrm{C}^{N}:|z-a|<r\right\}$.

2.1. Weight functions. A function $\omega: \mathbf{R} \rightarrow[0, \infty[$ is called a weight function if it is continuous, even, increasing on $[0, \infty[$, and if it satisfies $\omega(0)=$ $0, \omega(1)>0$, and also the following conditions:

( $\alpha) \omega(2 t)=O(\omega(t))$ as $t$ tends to infinity.

$(\beta) \omega(t)=O(t)$ as $t$ tends to infinity.

$(\gamma) \log (t)=o(\omega(t))$ as $t$ tends to infinity.

( $\delta) \phi: t \mapsto \omega\left(e^{t}\right)$ is convex.

A weight function $\omega$ is called non-quasianalytic, if it satisfies

$$
\int_{1}^{\infty} \frac{\omega(t)}{t^{2}} d t<\infty
$$

Otherwise it is called quasianalytic.

The radial extension $\tilde{\omega}$ of a weight function $\omega$ is defined as

$$
\tilde{\omega}: \mathrm{C}^{n} \rightarrow[0, \infty[, \quad \tilde{\omega}(z):=\omega(|z|) .
$$

It will also be denoted by $\omega$ in the sequel, by abuse of notation. The Young conjugate of the function $\phi=\phi_{\omega}$, which appears in $(\delta)$, is defined as

$$
\phi^{*}(x):=\sup \{x y-\phi(y): y>0\} .
$$

EXAMPLE 2.2. The following functions are weight functions (possibly after a change on the interval $[-\delta, \delta]$, for suitable $\delta>0$ ).

(1) $\omega(t):=|t|^{\alpha}, 0<\alpha<1$.

(2) $\omega(t):=\log (1+|t|)^{\beta}, \beta>1$.

(3) $\omega(t):=|t|(\log (1+|t|))^{-\beta}, \beta>0$.

(4) $\omega(t):=|t|$.

The weight function in (3) is quasianalytic for $\beta \in] 0,1]$ and non-quasianalytic for $\beta>1$. The weight function in (4) is also quasianalytic. 
2.3. Spaces of ultradifferentiable functions. Let $\omega$ be a given weight function. For a compact subset $K$ of $\mathrm{R}^{N}$ and $m \in \mathrm{N}$ denote by $C^{\infty}(K)$ the space of all $C^{\infty}$-Whitney jets on $K$ and define

$$
\begin{aligned}
\mathscr{E}_{\{\omega\}}^{m}(K):=\left\{f \in C^{\infty}(K)\right. & :\|f\|_{K, m} \\
& \left.:=\sup _{x \in K} \sup _{\alpha \in \mathrm{N}_{0}^{N}}\left|f^{(\alpha)}(x)\right| \exp \left(-\frac{1}{m} \phi^{*}(m|\alpha|)\right)<\infty\right\} .
\end{aligned}
$$

For an open set $G$ in $\mathrm{R}^{N}$, define the space of all $\omega$-ultradifferentiable functions of Roumieu type on $G$ as

$$
\begin{aligned}
& \mathscr{E}_{\{\omega\}}(G):=\left\{f \in C^{\infty}(G): \text { For each } K \subset G\right. \text { compact } \\
& \text { there is } \left.m \in \mathrm{N} \text { so that }\|f\|_{K, m}<\infty\right\} .
\end{aligned}
$$

It is endowed with the topology given by the representation

$$
\mathscr{E}_{\{\omega\}}(G)=\operatorname{proj}_{\leftarrow K} \operatorname{ind}_{m \rightarrow} \mathscr{E}_{\{\omega\}}^{m}(K),
$$

where $K$ runs over all compact subsets of $G$.

Note that $\mathscr{E}_{\{\omega\}}(G)$ is a countable projective limit of (DFN)-spaces, which is ultrabornological, reflexive and complete. If $\omega$ is non-quasianalytic this follows from Braun, Meise, Taylor [7], Proposition 4.9. If $\omega$ is quasianalytic, this follows from Rösner [16], Satz 3.25, together with Vogt [18], Theorem 3.4, and Wengenroth [19], Theorem 3.5. By $\mathscr{E}_{\{\omega\}}(G)^{\prime}$ we denote the dual of $\mathscr{E}_{\{\omega\}}(G)$ while $\mathscr{E}_{\{\omega\}}(G)_{b}^{\prime}$ denotes the strong dual. When $\omega$ is quasianalytic, the elements of $\mathscr{E}_{\{\omega\}}(G)^{\prime}$ are called quasianalytic functionals on $G$.

REMARK 2.4.

(a) If $\omega$ is the weight function $\omega(t)=|t|$, then the space $\mathscr{E}_{\{\omega\}}(G)$ coincides with the space $\mathscr{A}(G)$ of all real analytic functions on $G$. Martineau [11] proved that $\mathscr{A}(G)$ is ultrabornological.

(b) The classes of ultradifferentiable functions of Roumieu type in 2.3 are defined as in Braun, Meise, and Taylor [7].

2.5. Support functions. For a compact set $K$ in $\mathrm{R}^{N}, K \neq \emptyset$, the support function $h_{K}$ of $K$ is defined as

$$
h_{K}: \mathbf{R}^{N} \rightarrow \mathrm{R}, \quad h_{K}(\xi):=\sup _{x \in K}\langle x, \xi\rangle .
$$

Obviously, $h_{K}$ is a convex function which is positively homogeneous. 
2.6. Weighted spaces of holomorphic functions. Let $\omega$ be a weight function and $G$ an open convex subset of $\mathrm{R}^{N}$. Choose an increasing sequence $\left(K_{n}\right)_{n \in \mathrm{N}}$ of convex compact subsets of $G$ which satisfy the following conditions: $K_{n} \subset$ $\stackrel{\circ}{K}_{n+1}$ and $\bigcup_{n \in \mathrm{N}} K_{n}=G$. For $n \in \mathrm{N}$ denote by $h_{n}$ the support function of $K_{n}$ and define for $n \in \mathrm{N}, k \in \mathrm{N}$ the weights $v_{n, k} \in C\left(\mathrm{C}^{N}\right)$ by

$$
v_{n, k}(z):=\exp \left(-h_{n}(\operatorname{Im} z)-\frac{1}{k} \omega(z)\right) .
$$

The family $\left(v_{n, k}\right)_{n \in \mathrm{N}, k \in \mathrm{N}}$ is denoted by $\mathscr{V}_{\omega, G}$. Next denote by $H\left(\mathrm{C}^{N}\right)$ the space of all holomorphic functions on $\mathrm{C}^{N}$ and for a given non-negative upper semicontinuous function $v$ on $\mathrm{C}^{N}$ define the weighted semi-normed space

$$
H v:=\left\{f \in H\left(\mathrm{C}^{N}\right):\|f\|_{v}:=\sup _{z \in \mathrm{C}^{N}}|f(z)| v(z)<\infty\right\} .
$$

Then the weighted (LF)-space of entire functions associated with $\omega$ and $G$ is defined as

$$
\mathscr{V}_{\omega, G} H:=\operatorname{ind}_{n \rightarrow \operatorname{proj}_{\leftarrow k}} H v_{n, k} .
$$

2.7. Fourier-Laplace transform. Let $\omega$ be a weight function and $G$ a convex open set in $\mathrm{R}^{N}$. Then it is easy to check that for each $\mu \in \mathscr{E}_{\{\omega\}}(G)^{\prime}$, the FourierLaplace transform $\hat{\mu}$ of $\mu$, defined by

$$
\hat{\mu}(z):=\mu_{x}(\exp (-i\langle x, z\rangle)), \quad z \in \mathrm{C}^{N},
$$

(where $\langle x, z\rangle:=\sum_{j=1}^{N} x_{j} z_{j}$ ), is in $\mathscr{V}_{\omega, G} H$. In fact, the Fourier-Laplace transform

$$
\mathscr{F}: \mathscr{E}_{\{\omega\}}(G)_{b}^{\prime} \rightarrow \mathscr{V}_{\omega, G} H, \quad \mathscr{F}(\mu):=\hat{\mu},
$$

is a linear topological isomorphism. When $\omega$ is non-quasianalytic, this was shown in Braun, Meise, and Taylor [7], Theorem 7.4. When $\omega$ is quasianalytic this was shown by Meyer [14] when $N=1$ and in general by Rösner [16], Satz 2.19.

2.8. The projective hull $H \bar{V}_{\omega, G}$. For $\omega, G$, and $\mathscr{V}_{\omega, G}$ as in 2.6, we define the system $\bar{V}_{\omega, G}$ of weights associated with $\mathscr{V}_{\omega, G}$ according to Bierstedt, Meise and Summers [2], 0.2, as

$$
\begin{aligned}
\bar{V}_{\omega, G}:=\left\{\bar{v}: \mathrm{C}^{N} \rightarrow[0, \infty[: \bar{v} \text { is upper semi-continuous and for each }\right. \\
\left.n \in \mathrm{N} \text { there exist } \alpha_{n}>0 \text { and } k(n) \in \mathrm{N} \text { such that } \bar{v} \leq \alpha_{n} v_{n, k(n)}\right\} .
\end{aligned}
$$


Then the projective hull $H \bar{V}_{\omega, G}$ of $\mathscr{V}_{\omega, G} H$ is defined as

$$
H \bar{V}_{\omega, G}:=\operatorname{proj}_{\leftarrow \bar{v} \in \bar{V}_{\omega, G}} H \bar{v} .
$$

It is easy to check that $H \bar{V}_{\omega, G}$ is a complete locally convex space and that $\mathscr{V}_{\omega, G} H \subset H \bar{V}_{\omega, G}$ with continuous inclusion.

\section{Main results}

In this section we state and prove the main result of this paper which answers the following question in the negative: Is it possible to obtain the topology of the (LF)-space $\mathscr{V}_{\omega, G} H$ by the weighted sup-seminorms $\|\cdot\|_{\bar{v}}, \bar{v} \subset \bar{V}_{\omega, G}$ or in other words, is $\mathscr{V}_{\omega, G} H$ a topological subspace of its projective hull $H \bar{V}_{\omega, G}$ ? The precise result is stated in the following theorem.

THEOREM 3.1. For each weight function $\omega$ and for each convex open set $G$ in $\mathbf{R}^{N}$, the topology of $\mathscr{V}_{\omega, G} H$ is strictly finer than the one induced by its projective hull $H \bar{V}_{\omega, G}$.

REMark. For $\omega(t)=|t|$ and $G=\mathrm{R}^{N}$, Theorem 3.1 is due to Ehrenpreis [9]. For non-quasianalytic weight functions it was proved by the present authors in [4], Theorem 1. However, that proof uses the non-quasianalyticity of $\omega$ in two ways. First by the existence of non-trivial functions in $\mathscr{E}_{\{\omega\}}\left(\mathrm{R}^{N}\right)$ having compact support and second by the fact that $\omega$ admits a harmonic extension to the upper and lower half plane in C (see [7], sect. 2). Both facts are not available when $\omega$ is quasianalytic. Hence we have to use a new approach in this case.

REMARK. In [4] it was also shown that for non-quasianalytic weight functions $\omega$ the algebraic equality $\mathscr{V}_{\omega, G} H=H \bar{V}_{\omega, G}$ holds for each convex open set in $\mathrm{R}^{N}$. For quasianalytic weight functions this question will be investigated in Bonet, Meise, and Melikhov [6], where the research of [5] is continued.

The proof of Theorem 3.1 will be given only at the end of this section since we need to provide several auxiliary results first. A main point in our proof is the following lemma.

LEMMA 3.2. Let $G$ be an open convex set in $\mathrm{R}^{N}$ and let $\omega$ and $\sigma$ be weight functions which satisfy $\sigma(t)=o(\omega(t))$ as $t$ tends to infinity. Assume that $F \in A\left(\mathrm{C}^{N}\right)$ satisfies the following two conditions:

(1) There is $C \geq 1$ such that $|F(z)| \leq C \exp (C \sigma(z))$ for $z \in C^{N}$.

(2) There are $R>0$ and $D \geq 1$ such that for each $z \in C^{N},|z| \geq R$, there exists $w \in C^{N}$ such that

$$
|z-w| \leq D \sigma(z) \quad \text { and } \quad|F(w)| \geq \frac{1}{D} \exp (-D \sigma(z)) .
$$


Then the multiplication operator

$$
M_{F}: \mathscr{V}_{\omega, G} H \rightarrow \mathscr{V}_{\omega, G} H, \quad M_{F}(f):=F f,
$$

is an injective topological homomorphism if $\mathscr{V}_{\omega, G} H$ is endowed with the topology induced by $H \bar{V}_{\omega, G}$.

PRoof. It is easy to check that $M_{F}$ is injective, linear, and continuous for the topology induced by $H \bar{V}_{\omega, G}$. Hence it remains to show that the division map $F f \mapsto f$ is continuous for this topology. To do so, it is no restriction to assume that the existing constants $C$ and $D$ in (1) and (2) are both equal to $C$. Let $R$ as in (2). Since $\sigma=o(\omega)$ there is $L>0$ such that $\sigma(z) \leq|z|+L$, $z \in \mathrm{C}^{N}$. If $z, \zeta \in \mathrm{C}^{N}$ satisfy $|z-\zeta| \leq 4 C \sigma(z)$, then

$$
|\zeta| \leq|z|+4 C \sigma(z) \leq(4 C+1)|z|+4 C L,
$$

hence $\sigma(\zeta) \leq T \sigma(z)+T$ for some $T \geq 1$ depending only on $\sigma$. Therefore, we can apply condition (1) to conclude the existence of $A>0$ and $B>0$ such that

$$
\sup _{|z-\zeta| \leq 4 C \sigma(z)}|F(\zeta)| \leq A \exp (B \sigma(z)) .
$$

Let $D:=B+2 C$ and fix $\bar{w} \in \bar{V}_{\omega, G}$. Without loss of generality, we may assume that $\bar{w}$ is continuous and strictly positive by [2], Proposition 0.2 . Then for each $l \in \mathbf{N}$ there are $\alpha_{l}>0$ and $k(l) \in \mathbf{N}$ such that, for each $z \in \mathrm{C}^{N}$,

$$
\bar{w}(z) \leq \alpha_{l} \exp \left(-h_{l}(\operatorname{Im} z)-\frac{1}{k(l)} \omega(z)\right)=\alpha_{l} v_{l, k(l)}(z) .
$$

We define

$$
\tilde{v}(z):=\sup _{|z-\zeta| \leq 4 C \sigma(\zeta)} \bar{w}(\zeta) \exp (D \sigma(\zeta))
$$

and show that $\tilde{v}$ can be estimated by a weight $w \in \bar{V}_{\omega, G}$. Since each function $h_{n}$ is convex and positively homogeneous, $h_{n}$ is subadditive on $\mathbf{R}^{N}$. Moreover, for each $n \in \mathrm{N}$, there is $H_{n} \geq 1$, such that $h_{n}(x) \leq H_{n}|x|$ for every $x \in \mathrm{R}^{N}$. Therefore, for each $z, \zeta \in \mathrm{C}^{N}$ with $|z-\zeta| \leq 4 C \sigma(\zeta)$, we have

$$
h_{n}(\operatorname{Im} z) \leq h_{n}(\operatorname{Im} \zeta)+4 H_{n} C \sigma(\zeta) .
$$

Moreover, since $\sigma(t)=o(t)$ there is $R_{0}>R$ with $\sigma(t) \leq t /(8 C)$ if $t \geq R_{0}$. We determine $R_{1}>R_{0}$ such that $|z| \geq R_{1}$ and $|\zeta-z| \leq 4 C \sigma(\zeta)$ imply $|\zeta| \geq R_{0}$. For $|z| \geq R_{1}$ and $|\zeta-z| \leq 4 C \sigma(\zeta)$ we then have

$$
|\zeta| \leq|\zeta-z|+|z| \leq 4 C \sigma(\zeta)+|z| \leq \frac{1}{2}|\zeta|+|z|, \quad \text { and hence }|\zeta| \leq 2|z| \text {. }
$$


Again by $\sigma=o(\omega)$, for each $n \in \mathrm{N}$, there is $L_{n}>0$ such that,

$$
\left(4 H_{n} C+D\right) \sigma(\zeta) \leq \frac{1}{2 k(n)} \omega(\zeta)+L_{n} \quad \text { for all } \quad \zeta \in \mathrm{C}^{N} .
$$

Next note that if $\zeta, z \in C^{N}$ satisfy $|\zeta-z| \leq 4 C \sigma(\zeta)$ interchanging $z$ and $\zeta$ we get from (3.1)

$$
|z| \leq(4 C+1)|\zeta|+4 C L .
$$

Therefore, condition $(\alpha)$ for the weight $\omega$ implies the existence of $K>0$ and $S \in \mathrm{N}$, depending only on $\omega$ such that

$$
\omega(z) \leq K(\omega((1+4 C)|\zeta|)+\omega(4 L C)+1) \leq S \omega(\zeta)+S .
$$

For $n \in \mathrm{N}$ and $\zeta, z \in \mathrm{C}^{N}$ as before and $|z| \geq R_{1}$ we now get from (3.2) and (3.3)

$$
\begin{aligned}
v_{n, k(n)}(\zeta) \exp (D \sigma(\zeta)) & =\exp \left(-h_{n}(\operatorname{Im} \zeta)-\frac{1}{k(n)} \omega(\zeta)+D \sigma(\zeta)\right) \\
& \leq \exp \left(-h_{n}(\operatorname{Im} z)-\frac{1}{k(n)} \omega(\zeta)+\left(4 H_{n} C+D\right) \sigma(\zeta)\right) \\
& \leq e^{L_{n}} \exp \left(-h_{n}(\operatorname{Im} z)-\frac{1}{2 k(n)} \omega(\zeta)\right) \\
& \leq e^{L_{n}} e^{\frac{1}{2}} \exp \left(-h_{n}(\operatorname{Im} z)-\frac{1}{2 S k(n)} \omega(z)\right) .
\end{aligned}
$$

If we now define $\beta_{n}:=\alpha_{n} e^{L_{n}+\frac{1}{2}}, l(n):=2 S k(n) \in \mathrm{N}$ then for $z \in \mathrm{C}^{N}$, $|z| \geq R_{1}$, and $n \in \mathrm{N}$ we have

$$
\tilde{v}(z) \leq \beta_{n} v_{n, l(n)}(z) .
$$

Since $\tilde{v}$ is bounded on the disc in $\mathrm{C}^{N}$ of center 0 and radius $R_{1}, \tilde{v}$ can be estimated by an element in $\bar{V}_{\omega, G}$, and we can select $w \in \bar{V}_{\omega, G}$ which is strictly positive and continuous such that $\tilde{v} \leq w$.

Now, the same argument given at the end of the proof of [3], Proposition 2.5 , permits us to use the condition (2) to show that there is $\epsilon>0$ such that, if $f \in \mathscr{V}_{\omega, G} H$ satisfies $\|F f\|_{w} \leq \epsilon$, then $\|f\|_{\bar{w}} \leq 1$. This completes the proof of the continuity of the division.

Remark 3.3. Assume that $\mu \in \mathscr{E}_{\{\omega\}}\left(\mathrm{R}^{N}\right)^{\prime}$ satisfies the following continuity estimate

$$
\text { For each } m \in \mathrm{N} \text { there exists } C_{m}>0 \text { such that }
$$

$$
|\mu(\phi)| \leq C_{m}\|\phi\|_{B\left(0, \frac{1}{m}\right), m} \text { for each } \phi \in \mathscr{E}_{\{\omega\}}\left(\mathrm{R}^{N}\right) .
$$


Then for each open set $G$ in $\mathrm{R}^{N}, \mu$ induces a convolution operator,

$$
T_{\mu}: \mathscr{E}_{\{\omega\}}(G) \rightarrow \mathscr{E}_{\{\omega\}}(G), \quad T_{\mu}(\phi):=\mu * \phi: x \mapsto \mu(\phi(x-\cdot)),
$$

which is linear and continuous. The adjoint operator $T_{\mu}^{t}$ of $T_{\mu}$ is given by

$$
T_{\mu}^{t}: \mathscr{E}_{\{\omega\}}(G)^{\prime} \rightarrow \mathscr{E}_{\{\omega\}}(G)^{\prime}, \quad T_{\mu}^{t}(v):=\check{\mu} * v,
$$

where $\check{\mu} * v(\phi)=v(\mu * \phi)$, while $\check{\mu}(\phi):=\mu(\check{\phi})$ and $\check{\phi}(x)=\phi(-x)$ for $\phi \in \mathscr{E}_{\{\omega\}}\left(\mathrm{R}^{N}\right), x \in \mathrm{R}^{N}$.

If $G$ is convex, then the Fourier-Laplace transform $\mathscr{F}: \mathscr{E}_{\{\omega\}}(G)_{b}^{\prime} \rightarrow \mathscr{V}_{\omega, G} H$ is a linear topological isomorphism by 2.7. Let $F:=\mathscr{F}(\check{\mu})=\hat{\check{\mu}}$ and define

$$
M_{F}: \mathscr{V}_{\omega, G} \rightarrow \mathscr{V}_{\omega, G}, \quad M_{F}(f):=F f .
$$

Then the following identity holds:

$$
\mathscr{F} \circ T_{\mu}^{t}=M_{F} \circ \mathscr{F} .
$$

The next proposition shows that the proof of Theorem 3.1 can be reduced to the construction of a convolution operator $T_{\mu}$ on $\mathscr{E}_{\{\omega\}}(G)$ which is not surjective.

Proposition 3.4. Let $G$ be an open convex subset of $\mathrm{R}^{N}$ and let $\omega$ be a weight function. Assume that there exists $\mu \in \mathscr{E}_{\{\omega\}}\left(\mathrm{R}^{N}\right)^{\prime}$ and there is a weight function $\sigma$ which satisfies $\sigma=o(\omega)$ such that $F:=\hat{\check{\mu}}$ satisfies the conditions (1) and (2) of Lemma 3.2. If the operator $T_{\mu}: \mathscr{E}_{\{\omega\}}(G) \rightarrow \mathscr{E}_{\{\omega\}}(G)$ is not surjective, then the topology induced by $H \bar{V}_{\omega, G}$ is strictly coarser than the topology of $\mathscr{V}_{\omega, G} H$.

Proof. Suppose that the two topologies coincide. By Lemma 3.2 the operator

$$
M_{F}: \mathscr{V}_{\omega, G} H \rightarrow \mathscr{V}_{\omega, G} H, \quad M_{F}(f):=F f
$$

is an injective topological homomorphism. According to (3.5), the operator

$$
T_{\mu}^{t}: \mathscr{E}_{\{\omega\}}(G)_{b}^{\prime} \rightarrow \mathscr{E}_{\{\omega\}}(G)_{b}^{\prime}
$$

is also an injective topological homomorphism. Since $\mathscr{E}_{\{\omega\}}(G)$ is reflexive, a direct application of the Hahn-Banach theorem implies that

$$
T_{\mu}: \mathscr{E}_{\{\omega\}}(G) \rightarrow \mathscr{E}_{\{\omega\}}(G)
$$

is surjective in contradiction to the hypothesis. 
Remark. For $N \geq 3$ it follows from Rösner [16] that there even exist partial differential operators $T_{\mu}$ which satisfy the hypotheses of Proposition 3.4. However, for $N=2$ no partial differential operator with this property exists. This and the fact that heavy machinery is needed to obtain Rösner's results is the reason why we are going to use convolution operators and an argument which reduces the general case to the case $N=1$.

Next we present a version of an example due to Braun, Meise and Vogt [8], 3.11, which is suitable to construct later the functional $\mu$ required in Proposition 3.4.

Lemma 3.5. Let $\omega$ be a weight function. There is a weight function $\sigma_{0}$, satisfying $\sigma_{0}(t)=o(\omega(t))$ as $t$ tends to infinity, and there is $F \in H(\mathrm{C})$ such that the following conditions are fullfilled:

(i) There is $C>0$ with $|F(z)| \leq C \exp \left(\sigma_{0}(z)\right), z \in \mathrm{C}$.

(ii) $V(F):=\{z \in C: F(z)=0\}$ consists of a sequence $\left\{a_{j}: j \in \mathrm{N}\right\}$ of simple zeros with $\operatorname{Im} a_{j} \geq 2$ for each $j \in \mathrm{N}$.

(iii) For each $n \in \mathbf{N}$ there are infinitely many $j \in \mathbf{N}$ with $\operatorname{Im} a_{j} / \omega\left(a_{j}\right)=1 / n$.

(iv) There is $\epsilon_{0}>0$ such that, if $w \in C \backslash \bigcup_{j=1}^{\infty} B\left(a_{j}, 1\right)$, then

$$
|F(w)| \geq \epsilon_{0} \exp \left(-\sigma_{0}(w)\right) .
$$

(v) There are $\epsilon_{0}>0$ and $K_{0}>0$ such that, if $w \in \mathrm{C}$ satisfies $1 \leq\left|w-a_{j}\right| \leq$ 2 for some $j \in \mathrm{N}$, then

$$
|F(w)| \geq \epsilon_{0} \exp \left(-K_{0} \sigma_{0}\left(a_{j}\right)\right) .
$$

Proof. Since $\log t=o(\omega(t))$ as $t$ tends to infinity, we select $\left(s_{j}\right)_{j}$ in $[2, \infty[$ such that

(a) $s_{j+1} \geq 4 s_{j}$ for all $j \in \mathrm{N}$, and

(b) $n(t):=\operatorname{card}\left\{j \in \mathrm{N} \mid s_{j} \leq t\right\}$ satisfies $n(t) \log t=o(\omega(t))$ as $t$ tends to infinity.

Then we apply [7], 1.7 and 1.8 (a), to find a weight function $\sigma_{0}$ such that $n(t) \log t=o\left(\sigma_{0}(t)\right)$ and $\sigma_{0}(t)=o(\omega(t))$ as $t$ tends to infinity. For each $j \in \mathrm{N}$, we select $a_{j} \in \mathrm{C}$ with $\left|a_{j}\right|=s_{j}$ such that condition (iii) in the statement and $\operatorname{Im} a_{j} \geq 2$ for $j \in \mathbf{N}$ are satisfied. As in [8], 3.11, we define

$$
F(z):=\prod_{j=1}^{\infty}\left(1-\frac{z}{a_{j}}\right), \quad z \in \mathrm{C},
$$


which is an entire function by Rudin [17], Theorem 15.6 and satisfies condition (ii). Proceeding as in [8], 3.11, if $|z| \geq s_{1}$,

$$
\log |F(z)| \leq n(|z|) \log |z|+\log 2+\frac{4}{9} .
$$

This implies condition (i) in the statement.

Next we show that condition (iv) holds. Since $\sigma_{0}$ is a weight function, there is $t_{1}>s_{1}$ such that if $t \geq t_{1}$, then, for some $K>0, \sigma_{0}(2 t) \leq K \sigma_{0}(t)$. Now select $t_{0}>t_{1}$ with $\log t \leq \frac{1}{4 K} \sigma_{0}(t)$. If $|z| \geq t_{0}$, we have

$$
1 \leq 2 \log (2|z|)+\frac{4}{9} \leq 4 \log (2|z|) \leq \frac{1}{K} \sigma_{0}(2|z|) \leq \sigma_{0}(z) .
$$

Now select $j_{0} \in \mathbf{N}$ with $s_{j_{0}}>t_{0}$. Suppose that $w \in \mathbf{C} \backslash \bigcup_{j=1}^{\infty} B\left(a_{j}, 1\right)$ satisfies $|w| \geq s_{j_{0}}$. Then there is $l \geq j_{0}$ such that $s_{l} \leq|w|<s_{l+1}$. Since $\left|w-a_{l}\right| \geq 1$ and $\left|w-a_{l+1}\right| \geq 1$, we can proceed as in [8], 3.11, to conclude

$$
\log |F(w)| \geq-2 \log (2|w|)-\frac{4}{9} \geq-\sigma_{0}(w) .
$$

This yields $|F(w)| \geq \exp \left(-\sigma_{0}(w)\right)$ if $|w| \geq s_{j_{0}}, w \notin \bigcup_{j=1}^{\infty} B\left(a_{j}, 1\right)$, which clearly implies condition (iv).

We suppose now that $1 \leq\left|w-a_{j}\right| \leq 2$ for some $j \geq j_{0}+1$. Either $s_{j-1} \leq|w|<s_{j}$ or $s_{j} \leq|w| \leq s_{j+1}$. In any case, we can proceed as above to get

$$
|F(w)| \geq \exp \left(-\sigma_{0}(w)\right) .
$$

Since $\left|w-a_{j}\right| \leq 2$, we apply [7], Lemma 1.2, to find $S>0$ and $K>0$ with $-\sigma_{0}(w) \geq-S-K \sigma_{0}\left(a_{j}\right)$. Therefore $|F(w)| \geq e^{-S} \exp \left(-K \sigma_{0}\left(a_{j}\right)\right)$. Since $F(w)$ does not vanish on

$$
\bigcup_{j=1}^{j_{0}+1}\left\{w \in \mathrm{C}: 1 \leq\left|w-a_{j}\right| \leq 2\right\},
$$

we can conclude (v).

Definition 3.6. Let $\alpha=\left(\alpha_{j}\right)_{j}, \beta=\left(\beta_{j}\right)_{j}$ be sequences of non-negative real numbers such that $\beta_{j} \rightarrow \infty$, as $j \rightarrow \infty$ and let $\left(r_{n}\right)_{n \in \mathrm{N}}$ be an increasing sequence tending to 1 or to $\infty$. For $k, n \in \mathbf{N}$, we set

$$
\begin{aligned}
\lambda(n, k) & :=\left\{x \in \mathrm{C}^{\mathrm{N}}:\|x\|_{n, k}:=\sum_{j=1}^{\infty}\left|x_{j}\right| \exp \left(r_{n} \alpha_{j}+\frac{1}{k} \beta_{j}\right)<\infty\right\}, \\
K(n, k) & :=\left\{x \in \mathrm{C}^{\mathrm{N}}:\|x\|_{n, k}:=\sup _{j \in \mathbb{N}}\left|x_{j}\right| \exp \left(-r_{n} \alpha_{j}-\frac{1}{k} \beta_{j}\right)<\infty\right\} .
\end{aligned}
$$


We put, for $n \in \mathrm{N}, \lambda_{n}(\alpha, \beta):=\operatorname{ind}_{k} \lambda(n, k)$, and we denote by $\Lambda(\alpha, \beta)$ the projective spectrum $\left(\lambda_{n}(\alpha, \beta), i_{n+1}^{n}\right)$, where $i_{n+1}^{n}$ is the inclusion map. Then let

$$
\lambda(\alpha, \beta):=\operatorname{proj}_{n} \operatorname{ind}_{k} \lambda(n, k), \quad \text { and } \quad K(\alpha, \beta):=\operatorname{ind}_{n} \operatorname{proj}_{k} K(n, k)
$$

and note that $\lambda(\alpha, \beta)$ is a countable projective limit of (DFN)-spaces, and $K(\alpha, \beta)$ is an (LF)-space. By Meise [12], 1.6, $\lambda(\alpha, \beta)$ is a complete Schwartz space and $\lambda(\alpha, \beta)_{b}^{\prime}$ coincides with $K(\alpha, \beta)$.

The following result follows from Braun, Meise, and Vogt [8], Proposition 3.7, and Vogt [18], Theorem 4.3. The basic facts about the functor $\operatorname{Proj}^{1}$ can be found in these articles.

Lemma 3.7. Let $\alpha=\left(\alpha_{j}\right)_{j}$ and $\beta=\left(\beta_{j}\right)_{j}$ be sequences of non-negative real numbers such that $\beta_{j} \rightarrow \infty$ as $j \rightarrow \infty$ and let $\left(r_{n}\right)_{n \in \mathrm{N}}$ be an increasing sequence tending to 1 or to $\infty$. The following conditions are equivalent:

(1) $\operatorname{Proj}^{1} \Lambda(\alpha, \beta)=\{0\}$,

(2) $\lambda(\alpha, \beta)$ is bornological (or barrelled),

(3) $K(\alpha, \beta)$ is a complete (or regular) (LF)-space,

(4) there exists $\delta>0$ such that the set of finite limit points of the set $\left\{\alpha_{j} / \beta_{j} \mid\right.$ $\left.j \in \mathrm{N}, \beta_{j} \neq 0\right\}$ is contained in $\{0\} \cup[\delta, \infty[$.

Lemma 3.8. Let $\omega$ be a weight function and let $F \in H(\mathrm{C})$ and $\sigma_{0}$ be as in Lemma 3.5. Suppose that $h_{n}: R \rightarrow[0, \infty[$ is of one of the following types

(a) $h_{n}(t)=\left(1-\frac{1}{n}\right)|t|$,

(b) $h_{n}(t)=n|t|$,

(c) $h_{n}(t)=\max \left(\frac{1}{n} t, n t\right)$.

Define $v_{n, k}(z):=\exp \left(-h_{n}(\operatorname{Im} z)-\frac{1}{k} \omega(z)\right), z \in \mathrm{C}$. We set $\left.G=\right]-1,1[, \mathbf{R}$ or $] 0, \infty\left[\right.$ in the cases $(a),(b)$ and $(c)$ respectively. Then $F \in \mathscr{V}_{\omega, \mathrm{R}} H$, and we have

(1) $M_{F}\left(\mathscr{V}_{\omega, G} H\right)=\left\{f \in \mathscr{V}_{\omega, G} H \mid f\left(a_{j}\right)=0\right.$ for each $\left.j \in \mathrm{N}\right\}$, hence this ideal is closed in $\mathscr{V}_{\omega, G} \mathrm{H}$, and

(2) the quotient $\mathscr{V}_{\omega, G} H / M_{F}\left(\mathscr{V}_{\omega, G} H\right)$ is isomorphic to $K(\alpha, \beta)$ with $\alpha=$ $\left(\left|\operatorname{Im} a_{j}\right|\right)_{j}, \beta=\left(\omega\left(a_{j}\right)\right)_{j}$ and $r_{n}=1-\frac{1}{n}$ in case $(a)$ and $r_{n}=n$ in cases (b) and (c).

Proof. The condition in Lemma 3.5 ensures that the function $F$ belongs to $\mathscr{V}_{\omega, \mathrm{R}} H$ and that the multiplication operator $M_{F}$ defines a continuous linear operator from $\mathscr{V}_{\omega, G} H$ into itself. We define

$$
\rho: \mathscr{V}_{\omega, G} H \rightarrow K(\alpha, \beta) \text { by } \rho(f):=\left(f\left(a_{j}\right)\right)_{j} .
$$


Observe that, since $\operatorname{Im} a_{j} \geq 2$ by Lemma 3.5 (ii), then $h_{n}\left(\operatorname{Im} a_{j}\right)=r_{n}\left|\operatorname{Im} a_{j}\right|$, $n, j \in \mathrm{N}$. Consequently, if $f \in \mathscr{V}_{\omega, G} H,\left(f\left(a_{j}\right)\right)_{j} \in K(\alpha, \beta)$, and the map $\rho$ is well defined and continuous. We claim that $\rho$ is surjective. To prove this, fix $n \in \mathrm{N}$ and $x=\left(x_{j}\right)_{j} \in \bigcap_{k \in \mathrm{N}} K(n, k)$. Since $\|x\|_{n, k}<\infty$ for each $k$, we can find a weight function $\sigma$ such that

$$
\sigma_{0}(t) \leq \sigma(t)=o(\omega(t)) \quad \text { as } \quad t \rightarrow \infty,
$$

and

$$
\left|x_{j}\right| \leq \exp \left(h_{n}\left(\operatorname{Im} a_{j}\right)+\sigma\left(a_{j}\right)\right), \quad j \in \mathbf{N} .
$$

Then fix $\phi \in \mathscr{D}(B(0,2))$ satisfying $\left.\phi\right|_{B(0,1)} \equiv 1$ and $\left|\frac{\partial \phi}{\partial \bar{z}}(z)\right| \leq L$ for each $z \in \mathrm{C}$ and some $L>0$ and define

$$
\tilde{f}(z):=\sum_{j=1}^{\infty} x_{j} \phi\left(z-a_{j}\right), \quad z \in \mathrm{C} .
$$

Clearly $\tilde{f}(z)=0$ if $z \in \mathrm{C} \backslash \bigcup_{j=1}^{\infty} B\left(a_{j}, 2\right)$. Then let

$$
v(z):=-\frac{\overline{F(z)}}{|F(z)|^{2}} \frac{\partial \tilde{f}}{\partial \bar{z}} .
$$

Obviously, $\left.v\right|_{B\left(a_{j}, 1\right)} \equiv 0$ for each $j \in \mathrm{N}$. For $1 \leq\left|z-a_{j}\right| \leq 2$ Lemma 3.5 (v) gives

$$
|v(z)| \leq \frac{1}{|F(z)|}\left|x_{j}\right| L \leq L \epsilon_{0}^{-1} \exp \left(h_{n}\left(\operatorname{Im} a_{j}\right)+\sigma\left(a_{j}\right)+K_{0} \sigma_{0}\left(a_{j}\right)\right) .
$$

This implies, for $C_{1}>0$, and some $S>0$, depending on the weight $\sigma$, that

$$
v(z) \leq C_{1} \exp \left(h_{n}(\operatorname{Im} z)+S \sigma(z)\right), \quad \text { if } \quad 1 \leq\left|z-a_{j}\right| \leq 2, \quad j \in \mathbf{N} .
$$

If we denote by $d \lambda$ the Lebesgue measure on $C=R^{2}$, we get

$$
\int_{C}\left(|v(z)| \exp \left(-h_{n}(\operatorname{Im} z)-(S+1) \sigma(z)\right)\right)^{2} d \lambda(z) \leq\left(C_{1} D\right)^{2},
$$

with $D^{2}:=\int_{\mathrm{C}} e^{-2 \sigma(z)} d \lambda(z)$. By Hörmander [10], 4.4.2, there exists $g \in$ $L_{\text {loc }}^{2}(\mathrm{C})$ such that $\bar{\partial} g=v$ and

$$
\int_{C}\left(|g(z)| \exp \left(-h_{n}(\operatorname{Im} z)-(S+2) \sigma(z)\right)\right)^{2} d \lambda \leq\left(C_{1} D\right)^{2},
$$

and $g \in C^{\infty}(\mathrm{C})$, since $v \in C^{\infty}(\mathrm{C})$. 
Then $f:=\tilde{f}+g F$ satisfies $\bar{\partial} f=0$, hence $f \in H(\mathrm{C})$. Moreover $f\left(a_{j}\right)=$ $\tilde{f}\left(a_{j}\right)=x_{j}$ for each $j \in \mathbf{N}$. Proceeding as in the estimate of $v(z)$ above, we have, for $z \in B\left(a_{j}, 2\right)$,

$$
|\tilde{f}(z)| \leq\left|x_{j}\right| \leq C_{1} \exp \left(h_{n}(\operatorname{Im} z)+S \sigma(z)\right),
$$

hence

$$
\int_{C}\left(|f(z)| \exp \left(-h_{n}(\operatorname{Im} z)-(S+3) \sigma(z)\right)\right)^{2} d \lambda(z)<\infty .
$$

Since $f$ is holomorphic, standard arguments now imply the existence of $S_{1}>0$ such that

$$
\sup _{z \in \mathrm{C}}|f(z)| \exp \left(-h_{n}(\operatorname{Im} z)-S_{1} \sigma(z)\right)<\infty .
$$

As $\sigma(t)=o(\omega(t))$ as $t \rightarrow \infty$, we conclude $f \in \mathscr{V}_{\omega, G} H$ and $\rho(f)=\left(x_{j}\right)_{j}$.

The spaces $\mathscr{V}_{\omega, G} H$ and $K(\alpha, \beta)$ are (LF)-spaces, therefore the map $\rho$ is a surjective homomorphism [13], 24.30, and $\mathscr{V}_{\omega, G} H / \operatorname{ker} \rho$ is isomorphic to $K(\alpha, \beta)$. It remains to show that $\operatorname{ker} \rho=M_{F}\left(\mathscr{V}_{\omega, G} H\right)$ which is property (1) in the statement. The inclusion $M_{F}\left(\mathscr{V}_{\omega, G} H\right) \subset \operatorname{ker} \rho$ is trivial. Suppose that $g \in \mathscr{V}_{\omega, G} H$ satisfies $g\left(a_{j}\right)=0$ for each $j \in \mathrm{N}$. Clearly, $f:=g / F$ is holomorphic. Then note that there are $m \in \mathrm{N}$ and a weight function $\tau$, $\sigma_{0}(t) \leq \tau(t)=o(\omega(t))$, as $t \rightarrow \infty$, such that

$$
|g(z)| \leq C \exp \left(h_{m}(\operatorname{Im} z)+\tau(z)\right), \quad z \in \mathrm{C} .
$$

We can apply the condition (iv) of Lemma 3.5 to conclude, for $z \in \mathrm{C} \backslash$ $\bigcup_{j=1}^{\infty} B\left(a_{j}, 1\right)$,

$$
|f(z)| \leq C^{\prime} \exp \left(h_{m}(\operatorname{Im} z)+S^{\prime} \tau(z)\right),
$$

for some $S^{\prime}>0$ and $C^{\prime}>0$. By the maximum principle applied to each $\overline{B\left(a_{j}, 1\right)}$, we conclude $f \in \mathscr{V}_{\omega, G} H$, as in Meise [12], proof of 2.3.

REMARK. The cases (a) and (b) of Lemma 3.8 were treated in Meyer [15], Theorem 2.8, for a larger class of functions; see also Meise [12], Lemma 2.5. Since the case (c) does not appear there and since the proof is simpler in the special case needed here, we decided to prove the lemma for the convenience of the reader.

Lemma 3.9. Let $\omega$ be a weight function. For each open interval $G$ in $\mathrm{R}$ there is $\mu \in \mathscr{E}_{\{\omega\}}(\mathrm{R})^{\prime}$ such that $F:=\hat{\check{\mu}}$ satisfies the conditions $(i)-(v)$ of Lemma 3.5 for a weight function $\sigma_{0}$, and such that the convolution operator $T_{\mu}$ is not surjective on $\mathscr{E}_{\{\omega\}}(G)$. 
Proof. By dilation and/or translation, we may assume that $G=]-1,1[$ or $G=\mathrm{R}$ or $G=] 0, \infty[$, and we can select a fundamental sequence of compact intervals in $G$ such that the support functions are of one of the types (a) or (b) or (c) in Lemma 3.8 respectively. Let $F \in H(\mathrm{C})$ and $\sigma_{0}$ be as in Lemma 3.5. By 2.7 there exists $\mu \in \mathscr{E}_{\{\omega\}}(\mathrm{R})^{\prime}$ such that $F=\hat{\tilde{\mu}}$. Suppose that $T_{\mu}$ is surjective on $\mathscr{E}_{\{\omega\}}(G)$. Proceeding as in Meise [12], 3.4, formulas (2), (3), it follows from Lemma 3.8 that

$$
\left(\operatorname{ker} T_{\mu}\right)_{b}^{\prime} \simeq \mathscr{V}_{\omega, G} H / M_{F}\left(\mathscr{V}_{\omega, G} H\right) \simeq K(\alpha, \beta) .
$$

Since $\operatorname{Proj}^{1} \mathscr{C}_{\{\omega\}}(G)=\{0\}$ (this is proved by Meyer [15] for $G=$ ]-1, 1[ or $\mathrm{R}$, and by Rösner [16]) and since $T_{\mu}$ is surjective, it follows from Vogt [18], Theorem 1.5 (a result of Palamodov), that $\operatorname{Proj}^{1}\left(\operatorname{ker} T_{\mu}\right)=\{0\}$. Since $\lambda(\alpha, \beta)$ is semireflexive, and $\lambda(\alpha, \beta)_{b}^{\prime}=K(\alpha, \beta)$, we conclude $\operatorname{Proj}^{1} \Lambda(\alpha, \beta)=\{0\}$. This contradicts Lemma 3.7 by the choice of $\alpha, \beta$ and Lemma 3.5 (iii).

Lemma 3.10. Let $G \neq \emptyset$ be an open, convex, bounded subset of $\mathrm{R}^{N}, N \geq 2$. Then there exist a hyperplane $H$ and a parallel hyperplane $H_{+}$so that $G$ lies between $\mathrm{H}$ and $H_{+}$and such that there are points $a \in H \cap \partial G$ and $b \in H_{+} \cap \partial G$ for which the segment $] a, b[$ is contained in $G$.

Proof. Choose $a_{0}, a_{1} \in \bar{G}$ such that $\operatorname{diam} \bar{G}=\left|a_{0}-a_{1}\right|$. Let $H$ be the hyperplane which is orthogonal to the vector $a_{1}-a_{0}$ and which contains $a_{0}$, and let $H_{+}$be the parallel hyperplane through $a_{1}$. If the line $] a_{0}, a_{1}[$ lies in $G$, the lemma is proved. Otherwise, the convexity of $G$ implies that $\Delta_{1}:=$ convhull $\left(a_{0}, a_{1}\right) \subset \partial G$. Now apply the following induction argument: Assume that $a_{0}, \ldots, a_{k}$ are points in $\partial G$ for which

$$
\Delta_{k}:=\operatorname{convhull}\left(a_{0}, \ldots, a_{k}\right)
$$

has dimension $k$ (equiv. $a_{1}-a_{0}, \ldots, a_{k}-a_{0}$ are linearly independent). Choose a hyperplane $H_{k}$ which contains $\Delta_{k}$ and is a supporting hyperplane for $\bar{G}$. Since $\bar{G}$ is compact and convex, there is $a_{k+1} \in \partial G$ such that $\bar{G}$ is contained between $H_{k}$ and the hyperplane parallel to $H_{k}$ which contains $a_{k+1}$. Since $G \neq \emptyset$ and open, $a_{k+1} \notin H_{k}$. If there is a point $\xi \in \Delta_{k}$ such that the open segment ] $\xi, a_{k}[$ belongs to $G$, the lemma is proved. Otherwise

$$
\Delta_{k+1}:=\operatorname{convhull}\left(a_{0}, \ldots, a_{k+1}\right)
$$

has dimension $k+1$ and we can apply the induction step again. When $k=N$, the existence of $\xi \in \Delta_{N-1}$ with $] \xi, a_{N}[\subset G$ is obvious. Hence the proof is complete. 
LEMMA 3.11. Let $G$ be an open convex set in $\mathrm{R}^{N}, N \geq 2$, which is unbounded and is not equal to $\mathrm{R}^{N}$. Then $G$ satisfies one of the following two alternative conditions, up to a linear change of coordinates and up to a translation:

(1) $G \subset\left\{x \in \mathrm{R}^{N}: x_{1}>0\right\}$ and $\{(t, 0, \ldots, 0): t>0\} \subset G$.

(2) There exists $k$ with $1 \leq k \leq N-1$ and a bounded open convex set $G_{k}$ in $\mathrm{R}^{k}$, such that $G=G_{k} \times \mathrm{R}^{N-k}$.

PRoof. It is no restriction to assume $0 \in G$. Then define

$$
\phi: S^{N-1} \rightarrow[0, \infty], \quad \phi(\xi):=\sup \{t>0: t \xi \in G\} .
$$

Since $G$ is unbounded, the set

$$
M_{\infty}:=\left\{\xi \in S^{N-1}: \phi(\xi)=\infty\right\}
$$

is not empty.

Case 1. There is $\xi \in M_{\infty}$ such that $-\xi \notin M_{\infty}$. Then $\phi(-\xi)(-\xi) \in \partial G$. Let $H$ be a supporting hyperplane for $\bar{G}$ at this point. Then it is easy to see that after a translation and a linear change of variables we have (1).

Case 2. For each $\xi \in M_{\infty}$ also $-\xi \in M_{\infty}$. Then span $M_{\infty} \subset G$. Choose coordinates so that

$$
\operatorname{span} M_{\infty}=\left\{x \in \mathbf{R}^{N}: x_{1}=0, \ldots, x_{k}=0\right\},
$$

i. e., $\operatorname{dim} \operatorname{span} M_{\infty}=N-k$. Denote by $L:=\left\{x \in \mathrm{R}^{N}: x_{j}=0, j=\right.$ $k+1, \ldots, N\}$ and let

$$
G_{k}:=G \cap L .
$$

Since $L \cap M_{\infty}=\emptyset, G_{k}$ is a bounded convex set and it is easy to check that now (2) holds.

Proof of Theorem 3.1. We first treat the special case that $G$ is an open convex subset of $\mathrm{R}^{N}$ for which there is an open interval $G_{1} \subset \mathrm{R}$ such that

$$
G \cap(\mathrm{R} \times\{0, \ldots, 0\})=\left\{(t, 0, \ldots, 0): t \in G_{1}\right\},
$$

and

$$
G \subset\left\{\left(x_{1}, \ldots, x_{N}\right) \in \mathrm{R}^{N}: x_{1} \in G_{1}\right\} .
$$

By Lemma 3.9, we find $\mu \in \mathscr{E}_{\{\omega\}}^{\prime}(\mathrm{R})$ such that $T_{\mu}$ is not surjective on $\mathscr{E}_{\{\omega\}}\left(G_{1}\right)$ and that $\hat{\tilde{\mu}}$ satisfies the conditions (i)-(v) of Lemma 3.5. Define $v \in \mathscr{E}_{\{\omega\}}^{\prime}\left(\mathbf{R}^{N}\right)$ by

$$
\langle\nu, \phi\rangle:=\langle\mu, \phi(\cdot, 0, \ldots, 0)\rangle, \quad \phi \in \mathscr{E}_{\{\omega\}}\left(\mathbf{R}^{N}\right) .
$$


Then $\hat{v}(z)=\hat{\mu}\left(z_{1}\right)$ for each $z=\left(z_{1}, \ldots, z_{N}\right) \in \mathrm{C}^{N}$. Therefore it is easy to check that the choice of $\mu$ implies that $F:=\hat{\tilde{v}}$ satisfies the conditions (1) and (2) of Lemma 3.2. Hence it follows from Proposition 3.4 that the topology induced by $H \bar{V}_{\omega, G}$ is strictly coarser than the one of $\mathscr{V}_{\omega, G} H$, if we show that the convolution operator $T_{\nu}: \mathscr{E}_{\{\omega\}}(G) \rightarrow \mathscr{E}_{\{\omega\}}(G)$ is not surjective. To do so, assume that $T_{v}$ is surjective. To show that this assumption implies the surjectivity of $T_{\mu}$, let $\phi \in \mathscr{E}_{\{\omega\}}\left(G_{1}\right)$ be given. Then

$$
\tilde{\phi}(x):=\phi\left(x_{1}\right), \quad x=\left(x_{1}, \ldots, x_{N}\right) \in G
$$

is in $\mathscr{E}_{\{\omega\}}(G)$, due to the special form of $G$. Since we assume $T_{\nu}$ to be surjective, there is $\tilde{\psi} \in \mathscr{E}_{\{\omega\}}(G)$ satisfying $T_{\nu} \tilde{\psi}=\tilde{\phi}$. For $x \in G_{1}$ this implies

$$
\begin{aligned}
\phi(x) & =\tilde{\phi}(x, 0, \ldots, 0)=\left(T_{\nu} \tilde{\psi}\right)(x, 0, \ldots, 0)=v_{y}(\tilde{\psi}((x, 0, \ldots, 0)-y)) \\
& =\mu(\tilde{\psi}(x-\cdot, 0, \ldots, 0))=\left(T_{\mu} \psi\right)(x),
\end{aligned}
$$

where $\psi(x):=\tilde{\psi}(x, 0, \ldots, 0)$ for $x \in G_{1}$. Hence $T_{\mu}$ is surjective in contradiction to our choice of $\mu$. Therefore the theorem is proved in the special case.

To reduce the general case to the special case, let now $G$ be a non-empty open convex set in $\mathrm{R}^{N}$. Since $G=\mathrm{R}^{N}$ is certainly of the form treated in the special case, we may assume $G \neq \mathrm{R}^{N}$. Therefore it follows from Lemma 3.10 and 3.11 that modulo a translation we can find a hyperplane $H$ through the origin, and a real linear isomorphism $\Phi: \mathrm{R}^{N} \rightarrow \mathrm{R}^{N}$ such that

$$
\begin{aligned}
& \Phi(G) \subset\left\{y \in \mathrm{R}^{N}: 0<y_{1}<a\right\}, \quad a>0 \text { or } a=\infty, \\
& \Phi(H)=\left\{y \in \mathrm{R}^{N}: y_{1}=0\right\},
\end{aligned}
$$

and $\Phi(G)$ lies between $\Phi(H)$ and a parallel hyperplane or in a half space determined by $\Phi(H)$. Therefore $\Phi(G)$ is a convex open subset as we considered it in the special case.

Denote by $\Phi^{T}$ the real adjoint of $\Phi$, and find $d>0$ with

$$
\frac{1}{d}|x| \leq\left|\Phi^{T}(x)\right| \leq d|x|, \quad x \in \mathrm{R}^{N} .
$$

If $\left(K_{n}\right)_{n}$ is a fundamental sequence of convex compact subsets of $G$, then $\left(\Phi\left(K_{n}\right)\right)_{n}$ is a fundamental sequence of convex compact subsets of $\Phi(G)$. Moreover, for each compact set $K$ in $\mathrm{R}^{N}$

$$
h_{\Phi(K)}(y)=h_{K}\left(\Phi^{T}(y)\right), \quad y \in \mathbf{R}^{N} .
$$


Next define

$$
X: H\left(\mathrm{C}^{N}\right) \rightarrow H\left(\mathrm{C}^{N}\right), \quad X(g)(z):=g\left(\Phi^{T}(z)\right), \quad z \in \mathrm{C}^{N},
$$

where $\Phi^{T}$ is a real linear map canonically defined on $C^{N}$. We show that $X$ induces a linear topological isomorphism from $\mathscr{V}_{\omega, G} H$ onto $\mathscr{V}_{\omega, \Phi(G)} H$. Indeed, since $\left(\Phi^{-1}\right)^{T}=\left(\Phi^{T}\right)^{-1}$, only one estimate for the continuity is needed. Suppose that $g \in \mathscr{V}_{\omega, G} H$. Then there exists $n \in \mathrm{N}$ such that for each $k \in \mathrm{N}$ there is $C_{k}>0$ such that

$$
|g(z)| \leq C_{k} \exp \left(h_{K_{n}}(\operatorname{Im} z)+\frac{1}{k} \omega(z)\right), \quad z \in \mathrm{C}^{N} .
$$

If $z \in \mathrm{C}^{N}$, and $k \in \mathrm{N}$, we have

$$
\begin{aligned}
|X(g)(z)|=\left|g\left(\Phi^{T}(z)\right)\right| & \leq C_{k} \exp \left(h_{K_{n}}\left(\operatorname{Im} \Phi^{T}(z)\right)+\frac{1}{k} \omega\left(\Phi^{T} z\right)\right) \\
& \leq C_{k}^{\prime} \exp \left(h_{\Phi\left(K_{n}\right)}(\operatorname{Im} z)+\frac{L}{k} \omega(z)\right),
\end{aligned}
$$

for some $L \in \mathrm{N}$ which depends only on $\omega$ and $d>0$.

It is easy to check that

$$
\bar{V}_{\omega, \Phi(G)}=\left\{\bar{v} \circ \Phi^{T}: \bar{v} \in \bar{V}_{\omega, G}\right\} .
$$

This implies that

$$
Y: H \bar{V}_{\omega, G} \rightarrow H \bar{V}_{\omega, \Phi(G)}, \quad Y(g)(z):=X(g)(z)=g\left(\Phi^{T}(z)\right), \quad z \in \mathrm{C}^{N}
$$

is a linear topological isomorphism, too. If $j_{G}: \mathscr{V}_{\omega, G} H \rightarrow H \bar{V}_{\omega, G}$ denotes the inclusion map then we obviously have

$$
Y \circ j_{G}=j_{\Phi(G)} \circ X \quad \text { and hence } \quad j_{\Phi(G)}=Y \circ j_{G} \circ X^{-1} .
$$

By the special case $j_{\Phi(G)}$ is not a topological homomorphism, therefore $j_{G}$ cannot be a topological homomorphism. Thus we reduced the general case to the special case.

\section{REFERENCES}

1. Bierstedt, K. D., A survey of some results and open problems in weighted inductive limits and projective description for spaces of holomorphic functions, Bull. Soc. Roy. Sci. Liège 70 (2001), 167-182. 
2. Bierstedt, K. D., Meise, R., and Summers, W. H., A projective description of weighted inductive limits, Trans. Amer. Math. Soc. 272 (1982), 107-160.

3. Bonet, J., Galbis, A., and Meise, R., On the range of convolution operators on non-quasianalytic ultra-differentiable functions, Studia Math. 126 (1997), 171-198.

4. Bonet, J., and Meise, R., Ultradistributions of Roumieu type and projective descriptions, J. Math. Anal. Appl. 255 (2001), 122-136.

5. Bonet, J., Meise, R., and Melikhov, S. N., Holomorphic functions on locally closed convex sets and projective descriptions, Bull. Belg. Math. Soc. Simon Stevin 10 (2003), 491-503.

6. Bonet, J., Meise, R., and Melikhov, S. N., Projective representations of spaces of quasianalytic functionals, preprint 2003.

7. Braun, R. W., Meise, R., and Taylor, B. A., Ultradifferentiable functions and Fourier analysis, Resultate Math. 17 (1990), 206-237.

8. Braun, R. W., Meise, R., and Vogt, D., Existence of fundamental solutions and surjectivity of convolution operators on classes of ultra-differentiable functions, Proc. London Math. Soc. 61 (1990), 344-370.

9. Ehrenpreis, L., Solution of some problem of division. Part IV. Invertible and elliptic operators, Amer. J. Math. 82 (1960), 522-588.

10. Hörmander, L., Introduction to Complex Analysis in Several Variables, North-Holland, Amsterdam 1990.

11. Martineau, A., Sur la topologie des espaces des fonctions holomorphes, Math. Ann. 163 (1966), 62-88.

12. Meise, R., Sequence space representations for zero-solutions of convolution equations on ultradifferentiable functions of Roumieu type, Studia Math. 92 (1989), 211-230.

13. Meise, R., and Vogt, D., Introduction to Functional Analysis, Oxford Univ. Press 1997.

14. Meyer, T., Die Fourier-Laplace Transformation quasianalytischer Funktionale und ihre Anwendung auf Faltungsoperatoren, Diplomarbeit, Düsseldorf 1989.

15. Meyer, T., Surjectivity of convolution operators on spaces of ultradifferentiable functions of Roumieu type, Studia Math. 125 (1997), 101-129.

16. Rösner, T., Surjektivität partieller Differentialoperatoren auf quasianalytischen RoumieuKlassen, Dissertation, Düsseldorf 1997.

17. Rudin, W., Real and Complex Analysis, 3rd Ed. McGraw-Hill, New York 1987.

18. Vogt, D., Topics on projective spectra of $(L B)$-spaces, in "Advances in the theory of Fréchet spaces" (Ed. T. Terzioglu), NATO Advanced Science Institutes, Series C, 287 (Kluwer, Dordrecht 1989), 11-27.

19. Wengenroth, J., Acyclic inductive spectra of Fréchet spaces, Studia Math. 120 (1996), 247258.

DEPARTAMENTO DE MATEMÁTICA APLICADA

UNIVERSIDAD POLITÉCNICA DE VALENCIA

E-46071 VALENCIA

SPAIN

E-mail: jbonet@mat.upv.es
MATHEMATISCHES INSTITUT

HEINRICH-HEINE-UNIVERSITÄT

UNIVERSITÄTSSTRAßE 1

D-40225 DÜSSELDORF

GERMANY

E-mail: meise@cs.uni-duesseldorf.de 